\title{
Scale Models Formulation of Switched Reluctance Generators for Low Speed Energy Converters
}

This paper is a postprint of a paper submitted to and accepted for publication in IET Electric Power Application Journal and is subject to Institution of Engineering and Technology Copyright. The copy of record is available at IET Digital Library.

P. Lobato, J.A.Dente, J. Martins, A.J. Pires - IET Electric Power Application Journal; vol.9, n.9, pp.652-659, November 2015. ISSN 1751-8660. DOI: 10.1049/iet-epa.2014.0451 


\title{
Scale Models Formulation of Switched Reluctance Generators for Low Speed Energy Converters
}

\author{
P. Lobato*(‥A.), J.A.Dente**, J.F. Martins***, A. J. Pires* \\ * Department of Electrical Engineering, ESTSetúbal, Polytechnic Institute of Setúbal/CTS-UNINOVA \\ Campus do IPS Rua Vale de Chaves Estefanilha, 2910-761 Setúbal, Portugal (tel. +351 265790000 ) \\ e-mail: pedro.lobato@estsetubal.ips.pt; armando.pires@estsetubal.ips.pt \\ ** Instituto Superior Técnico, Lisboa, Portugal \\ e-mail: edentepc@ist.utl.pt \\ *** Department of Electrical Engineering, FCT, New University of Lisbon/CTS-UNINOVA \\ Campus de Caparica, 2829-516 Caparica, Portugal \\ e-mail: jf.martins@fct.unl.pt
}

\begin{abstract}
This paper presents a design methodology based on scale models for low speed Switched Reluctance Generators (SRG). This work is motivated by the application of SRG to direct-drive wind turbines and other low speed renewable energy systems. In direct drive energy converters considerable simplifications result from the elimination of the gear box which has generally been used to interface a slowly rotating shaft with the generator shaft. The comparison and evaluation of magnetic structures plays an important role in the SRG design. General design methodologies are usually oriented towards the evaluation of stator/rotor poles combinations for regular switched reluctance machines. Besides covering that feature, the formulation of scale laws proposed is also suitable to compare other SRG topologies distinguished by different characteristics of electric and magnetic circuits and their own relative position. In addition, this methodology can be extended to other physical phenomena like thermal changes and magnetic saturation by introducing some constraints. The running example compares a modular short flux-path topology versus a low speed $20 \mathrm{~kW}$ prototype SRG designed for a direct drive wind turbine. The modular topology can optimize the efficiency and weight taking benefits from the significant gain of power per unit of volume and lower losses.
\end{abstract}

Keywords - Scale Models Methodology, Similarity Laws, Low Speed Energy Converters, Switched Reluctance Generator, SRG Design.

\section{NOMENCLATURE}

$\begin{array}{ll}\boldsymbol{A}_{\boldsymbol{S}} & \text { Area of the material surface exposed for cooling purposes. } \\ \boldsymbol{B} & \text { Magnetic flux density. } \\ \boldsymbol{h} & \text { Machine core length. } \\ \boldsymbol{h}_{\boldsymbol{e}} & \text { Equivalent heat transfer coefficient. } \\ \boldsymbol{H} & \text { Magnetic field. } \\ \boldsymbol{i} & \text { Instantaneous phase current. } \\ \boldsymbol{I} & \text { Peak amplitude of fundamental harmonic of phase current. }\end{array}$




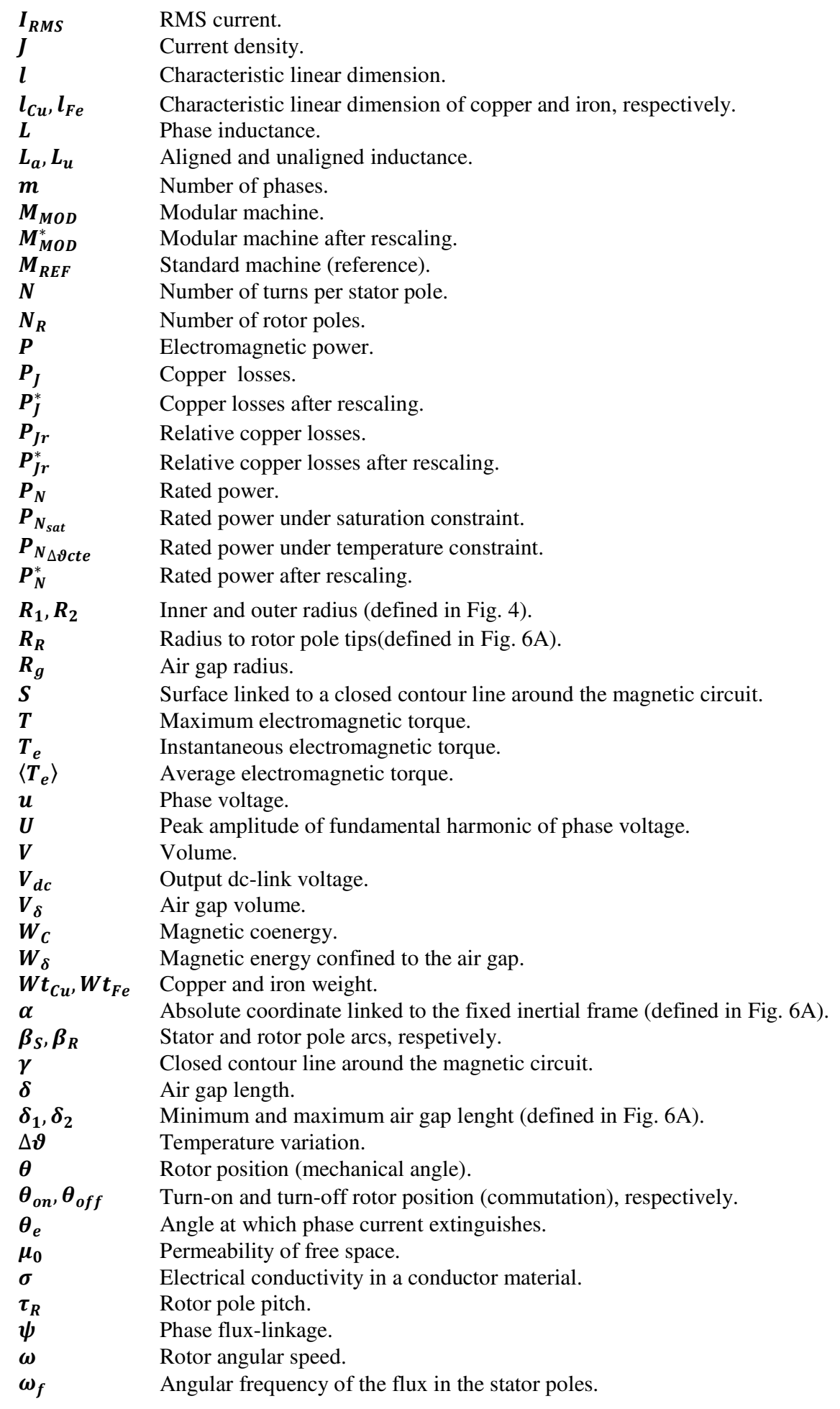

\section{INTRODUCTION}

Several aspects regarding the switched reluctance (SR) drives development have been a subject of research and further improvements for some decades and also in the recent past [1-5]. For example, the control techniques, loss optimization, torque ripple and acoustic noise illustrate the progress in knowledge regarding SR machines. The operation of the SR generator (SRG) has also deserved some attention, although not as much as the operation of the motor [6- 
10]. The SRG research work has been mainly directed at the automotive, aerospace and wind energy domains [11-17].

Previous and older treatments of SRG have focused on the design of high-speed SRG (see [18] and the references cited therein), which mainly rely on the regular magnetic structures [19] of two opposing stator poles per phase and a minimal number of rotor poles.

The SR machine is inherently a variable speed drive that can be easily controlled and matched to its load by controlling the instants of energizing and de-energizing the stator phases [20]. Furthermore, robustness and simple construction (only concentrated coils on the stator), control flexibility, high power density, high fault tolerance and good efficiency in a wide speed range [21-22], make this machine suitable for direct drive energy converters and attractive for harsh environmental applications [23-26].

Over the last decades more research efforts have been carried out regarding the use of SRG in direct drive wind energy systems. Though there are different magnetic circuit topologies, SRG design methodologies generally aim to choose the number of stator and rotor poles for regular topologies [27-28]. The formulation of similarity (or scale) laws [29] proposed in this paper provides the tools to compare other SRG topologies differentiated by diverse characteristics of electric and magnetic circuits and their own relative position. In addition, the use of this scale models methodology makes it easy to incorporate in the system analysis other physical phenomena, such as thermal effect and magnetic saturation, by introducing some constraints. Thus, this work underlines dimensional and similarity arguments that extend previous discussions about SRG design into a more general context.

This work is motivated by the application of SRG to direct-drive wind turbines and other low speed renewable energy systems. Direct drive energy converters benefit greatly from the elimination of the gearbox, which has traditionally been used to interface a slowly prime mover shaft with the generator shaft. In fact the gearbox is a considerable cost component that reduces the system reliability and its overall efficiency. Moreover, the actual trend of exploiting the offshore wind resources makes robustness and reliability vital to the economic operation of wind turbines in that specific environment.

In this paper the scale models methodology is applied to the SRG design by evaluating and comparing a modular short-flux path topology with a $20 \mathrm{~kW}$ regular SRG prototype operating at a rated speed in the region of $100 \mathrm{rpm}$ for use in a direct drive wind turbine [30]. To achieve that objective, together with the dimensional analysis, a field-based model is developed and a rescaling operation is performed.

This paper introduces a simple and already known dimensional similarity-based methodology ready to be used as a design tool for topologies comparison and discussion on SRG constructive paradigms. 


\section{SCALE MODELS METHODOLOGY FOR SRG}

\subsection{Energy Conversion}

A classic 4-phase regular topology with 8 stator poles and 6 rotor poles $(8 / 6)$ is shown in Fig.1-A, where $\beta_{r}$ and $\beta_{s}$ represent the rotor and stator pole arcs.

Neglecting saturation phenomena at this point, Fig. 1-B shows the idealized inductance $\boldsymbol{L}$, phase flux-linkage $\boldsymbol{\psi}$, phase current $\boldsymbol{i}$ and voltage $\boldsymbol{u}$ profiles of a SRG for single-pulse operation where $V_{S}$ is the dc-link voltage and $L_{a}, L_{u}$ are the aligned inductance and the unaligned inductance respectively. In a regular SR machine with $\boldsymbol{N}_{\boldsymbol{r}}$ rotor poles those waveforms are periodic in $\tau_{R}$, that is the rotor pole-pitch $\left(2 \pi / N_{r}\right)$.

During the excitation period, from $\theta_{o n}$ to $\theta_{\text {off }}$, current flows from the supply and energy is stored in the machine magnetic field. After commutation, at $\theta_{\text {off }}$, the excitation energy is returned to the supply while the energy provided by the prime mover, is converted into electrical energy.

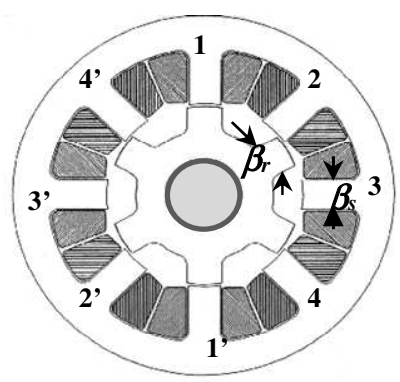

Fig1-A

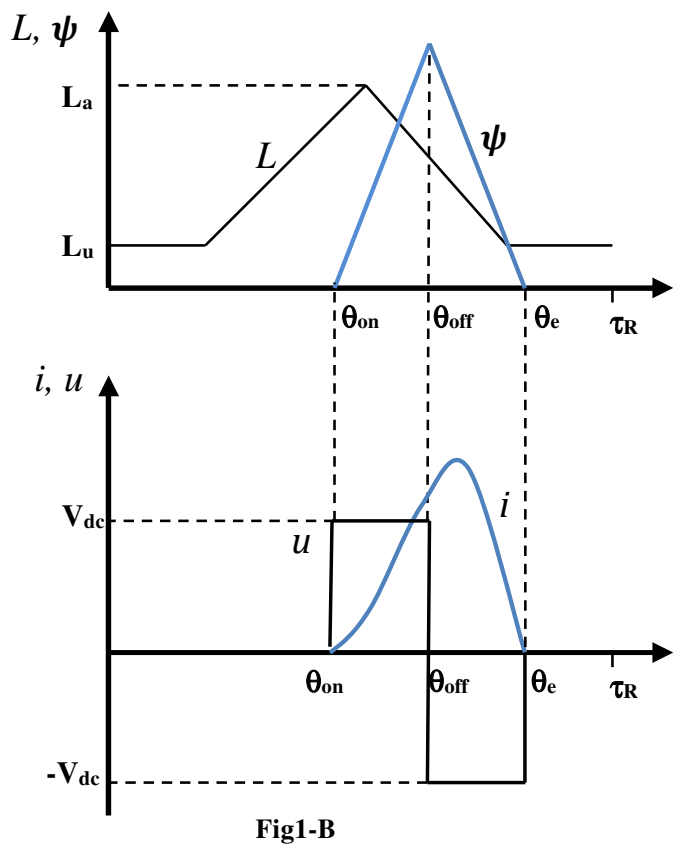

Fig. 1 - A. Cross-section of a regular four-phase 8/6 SR topology.

B. Idealized inductance profile and waveforms of a single-pulse controlled SRG. 
At $\theta_{e}$ all the flux from excitation period has been extinguished and no more electrical energy is returned to the supply. If losses are neglected then the output energy, over each stroke, exceeds the excitation energy by the mechanical energy supplied.

The SR machine is capable of operating continuously as a generator if the electrical output energy exceeds the excitation energy, i.e., by keeping the excitation period so that the bulk of the winding conduction period comes after the aligned position $(d L / d \theta<0)$. The SRG can be operated in single pulse mode over a wide speed range, which results in low switching losses [21].

\subsection{Scale Laws and Design Considerations}

In regards to SR machines design, there are a multiplicity of feasible topologies differentiated by the properties of the electrical and magnetic circuits and their relative location. However, in spite of that diversity, there are intrinsic constraints related to those machines which affect their dimensions and performance. Similarly to other conventional electromechanical rotating systems, the SRG operation is sustained by the magnetic field. The electromagnetic linkage of electrical and magnetic circuits is by itself a constraint. That linkage, between magnetic field intensity $\boldsymbol{H}$ and current density $\boldsymbol{J}$, is well represented by Ampère's law in integral form:

$$
\oint_{\gamma} H \cdot d l=\int_{S} J \cdot d S
$$

Characteristic dimensions of electrical and magnetic circuits are implicit in Ampère's law. Assuming that the machine design is oriented towards a scale change, the equation (1) can be written in the form of (2) where $\boldsymbol{l}$ represents a characteristic linear dimension.

$$
B \propto J
$$

This proportional relationship underlines that current excited magnetic circuits impose a scale-factor in their linear dimensions, which is reflected in the relationship / $\boldsymbol{J}$. Hence, significant changes of performance and machine features can be achieved by modifying that characteristic linear dimension.

Other constraints such as power supplied, losses, efficiency and temperature also determine the machine design. The following subsections present the scale relationships involving some of the variables mentioned and the machine dimensions with the aim of comparing and predicting certain SRG dimensional characteristics and parameters based on scale models. 


\subsubsection{Rated power}

In order to attain the aim pursued, a regular SR machine will be considered, as shown in Fig.1-A. These classical topologies are characterized by the symmetry of both rotor and stator poles about their centre-lines and equally spaced around the rotor and stator, respectively. Each phase winding comprises two coils, wound on opposite poles. Once the winding consists of a number of electrically separate phases and the SRG behaves as a single-phase excited machine, a study per phase will be performed.

SR machines designs, for operation at low speeds, usually point to a significant number of poles. This design orientation can be easily understood by focusing in the generator operation mode, illustrated in Fig.1-B. Neglecting the voltage drop in the winding resistance, the voltage equation for each phase can be represented as:

$$
u \cong \frac{d \psi}{d t}
$$

Both phase voltage $\boldsymbol{u}$ and flux-linkage $\boldsymbol{\psi}$ waveforms, can be approximated by a Fourier series comprising a fundamental component and a series of lower peak amplitudes harmonics. Neglecting harmonics of higher orders the previous relationship can be treated in terms of RMS values of the respective fundamental components as expressed by:

$$
\boldsymbol{U} \propto \omega_{f} \Psi
$$

where $\boldsymbol{\omega}_{\boldsymbol{f}}$ is the angular frequency of the flux in the stator poles.

On the other hand, the angular frequency of the flux in the stator poles is proportional to the angular velocity of the rotor $\boldsymbol{\omega}$ and is therefore given by :

$$
\omega_{f}=N_{R} \omega
$$

where $\boldsymbol{N}_{\boldsymbol{R}}$ is the number of rotor poles.

Revisiting the relationship indicated by (2) and, as the average electrical power related to one phase is proportional to the product of the voltage and phase current, a scale law of rated power for SR machines can be expressed by

$$
P_{N_{1}} \propto U I \propto N_{R} \omega \psi I
$$


Taking into account the representation of the phase current in terms of current density one may write the proportional relationship by introducing the scale factor $\boldsymbol{l}$. Hence the rated power law of a m-phase SR machine is suitably expressed in the form of

$$
P_{N} \propto m N_{R} \omega\left(B l^{2}\right)\left(J l^{2}\right) \propto m N_{R} \omega B J l^{4}
$$

It should be noted that the criteria of selecting a greater or smaller number of variables to be explicit in the scale laws depend only on the characteristics and parameters considered relevant for the proposed comparison of topologies. For instance, the number of rotor poles is a significant parameter in evaluating low speed SRG designs since $B$ is limited by magnetic saturation of the iron and $J$ should be kept under certain limits due to copper losses and the preservation of the insulating material. Therefore a compromise involving the number of poles, dimensions related either with the magnetic circuit as electrical circuit and phase current is required. For that purpose particular design details of SR topologies related with the magnetic circuit drawing and the windings location will be investigated in order to increase the available inner space of the machine taking advantage of an improved air circulation. These aspects will be addressed in section 3 .

\subsubsection{Losses}

The Joule losses in a conductor material of volume $\boldsymbol{V}$ and electrical conductivity $\boldsymbol{\sigma}$ is given by

$$
P_{J}=\int_{V} \frac{J^{2}}{\sigma} d V
$$

Considering the overall losses due to Joule effect related with the electric circuit of each phase, the proportional relationship of copper losses of a m-phase SR machine is therefore expressed by

$$
P_{J} \propto m J^{2} l^{3}
$$

The prediction of SGR magnetic losses is not trivial due to the fact that different parts of the iron core are not subjected to the same frequency of magnetic flux. At a constant rotor speed, the period and form of the flux waveforms in the core differ from part to part. A detailed study presented in [19] on the flux harmonic spectra in different parts of the iron core also contributes towards understanding the SR machine noise. In addition, the fact that this machine operates in 
varying levels of saturation further complicates the estimation of iron core losses. Although, in general the mutual flux can be neglected in SR machines [31] the analysis complexity grows as the number of phases increases.

However, due to lower thermal inertia of the copper compared with the iron and the practical temperature limits of insulators, the iron losses are less critical in terms of machine design and will not be included in this dimensional analysis.

\subsubsection{Saturation and temperature constraints}

The previous relationships are suitable to observe some physical effects and limitations as well as to know how scale laws handle these when applied in SRG design. Assuming a reference machine that is supposed to be properly designed, the other machines are similar scale models, in which the laws of proportionality should respect the material constraints as set out below. In order to exemplify that feature, a SRG characterized by a rated power, losses, maximum flux and current densities, were considered. These reference values are assumed as base values allowing the comparison with similar machines built in a different scale by using per-unit (p.u.) quantities.

In the scale models methodology applied to electromagnetic devices there is one degree of freedom to select the variables $B$ and $J$ as both are interrelated by (2). Although the flux density is significantly different in diverse regions and instants, the saturation levels should be kept constant in all regions of the magnetic circuit. Therefore the practical limitation imposed by the magnetic saturation can be expressed in terms of scale laws by (10). Thereby, the rated power for the SRG assumes now the form of (11).

$$
\begin{gathered}
J \propto \frac{B}{l} \\
P_{N_{s a t}} \propto m N_{R} \omega B^{2} l^{3}
\end{gathered}
$$

At a certain stage of machine design the heating phenomena has to be considered. In effect the balance between the temperature increase and the proper temperature levels of insulating materials is critical for the machine lifetime. Under thermal stability conditions, for the reasons referred in subsection \&2.2.2, it is assumed that the temperature variation $\Delta \boldsymbol{\vartheta}$ is proportional to copper losses as indicated by (12). In this relationship the constant $\boldsymbol{h}_{\boldsymbol{e}}$ is an equivalent heat transfer coefficient for all heat exchanges and $\boldsymbol{A}_{\boldsymbol{S}}$ is the cooling surface area of materials involved. 


$$
\Delta \vartheta \propto \frac{P_{J}}{h_{e} A_{S}} \propto \frac{m J^{2} l^{3}}{l^{2}} \propto J^{2} l
$$

Once introduced the constraint of temperature as a criteria $\Delta \boldsymbol{\vartheta}$ should be kept constant. The current density is expressed by (13) which points out the lower current density in larger machines. Thereby, the scale relationship indicated for rated power can be written simply by (14).

$$
\begin{gathered}
J \propto \frac{1}{\sqrt{l}} \\
P_{N_{\Delta \vartheta} c t e} \propto m N_{R} \omega B l^{7 / 2}
\end{gathered}
$$

At this point it is possible to verify that there is a scale gain in the efficiency of SR machines e.g. larger SRG present lower relative losses than smaller machines. That said, the copper losses are given by (15) in which it was assumed that the flux density is kept constant to make full use of the iron properties. The relationship (13) was also used to ensure that temperature doesn't exceed the limit values of the isolating materials.

$$
P_{J r}=\frac{P_{J}}{P_{N}} \propto \frac{m J^{2} l^{3}}{m N_{R} \omega B l^{7 / 2}} \propto \frac{1}{N_{R} \omega B l^{3 / 2}}
$$

So far this design methodology has been developed on a geometrical-scale factor basis having additional constraints to fix $B$ and $J$ densities. Larger machines are easily driven into saturation because the flux density can achieve high levels with lower relative current densities. In opposition to smaller machines design strategy, in larger machines the emphasis should be placed on the circuit magnetic dimension and the decrease of the relative dimension of the conductive material. These results suggest a mutation of scales, thereby benefiting from the behaviors of $B$ and $J$ with respect to the rated power. That mutation of scales is accomplished through two distinct scales, one for copper and another for iron. Thus, having in mind a better use of the conductive material and the core iron, two specific dimensions of each material will be used, $\boldsymbol{l}_{\boldsymbol{C u}}$ e $\boldsymbol{l}_{\boldsymbol{F e}}$. Therefore the relationship (7) assumes the form of (16) and the relationship (2) is now written as (17). These two degrees of freedom correspond to an equal number of constraints in order to fix the flux and current densities.

$$
P_{N} \propto m N_{R} \omega\left(B l_{F e}{ }^{2}\right)\left(J l_{C u}{ }^{2}\right)
$$




$$
B l_{F e} \propto J l_{C u}^{2}
$$

The two degrees of freedom available are used to keep the density flux as well as the temperature variation constant. In such case the copper characteristic dimension plays a key role in limiting the temperature increase. The relationship (18) will now replace (13).

$$
J \propto \frac{1}{\sqrt{l_{C u}}}
$$

As the weight of the materials is proportional to the cube of the respective specific dimensions, the relationships (19) and (20) are considered representative of the copper and iron weights of the modules set, $\boldsymbol{W} \boldsymbol{t}_{\boldsymbol{F} \boldsymbol{e}}$ and $\boldsymbol{W} \boldsymbol{t}_{\boldsymbol{C u}}$. Figure 2 highlights that, in relative terms, the copper weight decreases with the rated power and the iron weight is directly proportional to the rated power.

$$
\begin{gathered}
W t_{F e} \propto P_{N} \\
W t_{C u} \propto P_{N}^{2 / 3}
\end{gathered}
$$

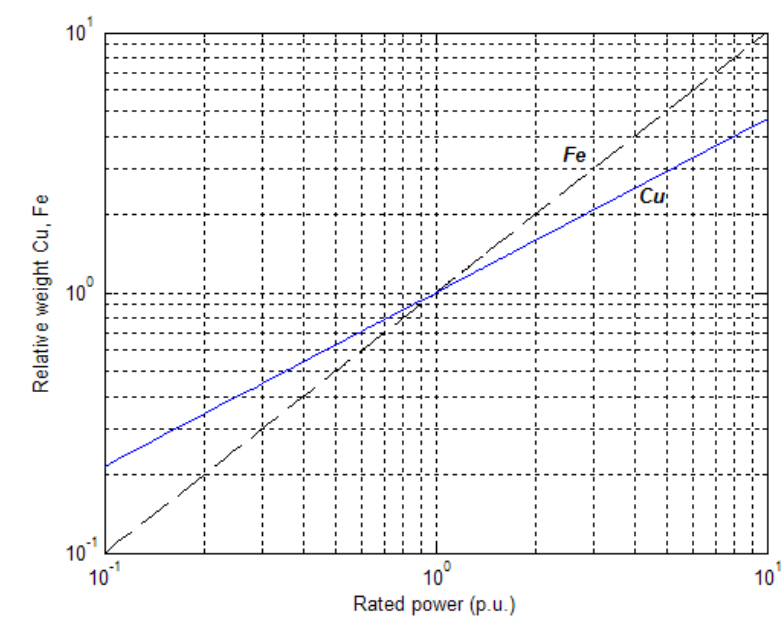

Fig. 2 - Relative weights of iron and copper keeping flux density and temperature increase both constant.

To fix both the variation of temperature and the flux density consist of not allowing the magnetic saturation and temperature materials limits to be exceeded. It is under these constraints that the scale relationship (21), reporting Joule losses at SR system is formulated. Fig. 3 shows 
that the relative losses increase as rated power decreases. That increase is steeper for lower rated powers.

$$
P_{J r} \propto P_{N}{ }^{-5 / 9}
$$

In spite of the temperature constraint, it is observed that, the scale gain effect persists for larger SR machines rather than smaller machines due to lower relative losses in the former case. In effect the relative copper losses are smaller for larger machines and they are higher for smaller machines due to current density that assume, in relative terms, smaller values in large SR machines as shown in Fig.3. In fact, the adopted scale criteria have shown an increase in current density which in turn causes higher copper losses. This increase of copper losses is not balanced by the flux density decrease.

The dimensional analysis performed so far makes it possible to predict that efficiency tends to increase in large SR machines and decrease in small ones. To counteract that trend in smaller machines, it is considered a good practice to increase, in relative terms, the linear dimension of the copper and decrease the dimension of the magnetic circuit.

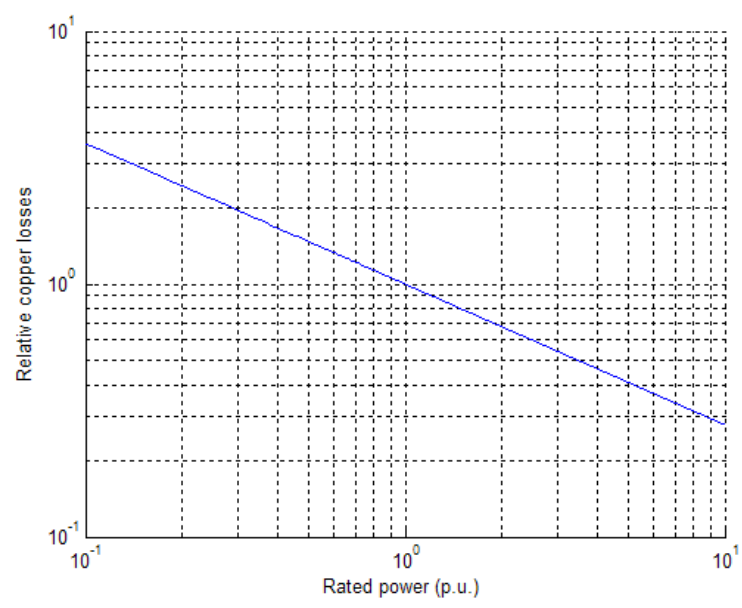

Fig. 3 - Relative copper losses with temperature increase and density flux both constant.

\section{SRG DESIGN}

\subsection{Short flux-path topology}

According to the scale criteria thus far, the previous evaluation of the copper weight and copper losses, clearly support the preference for larger machines over smaller machines. The selection of a magnetic structure that takes into account a shorter flux-path may reduce the magnetomotive force (MMF) absorbed in the iron core. A long flux-path of the regular SRG is 
illustrated in Fig.4-A and an example of a short flux-path is shown in Fig.4-B highlighting the flux closing between two adjacent rotor poles [32]. In order to compare the flux-path length $\boldsymbol{l}_{\boldsymbol{F} e, s f}$ of a short-flux path (SFP) topology with the length of the regular machine $\boldsymbol{l}_{\boldsymbol{F} \boldsymbol{e}}$, an exterior radius $\boldsymbol{R}_{\mathbf{2}}$ similar to both topologies is assumed. The circular arc length of the shaft contour is neglected for simplicity of analysis. Using the scale laws the effect on copper losses and weight can be evaluated in relative terms. The relationship of the flux paths is approached by (22).

$$
\frac{l_{F e, s f}}{l_{F e}} \cong \frac{\frac{\pi}{m}\left(R_{1}+R_{2}\right)+2\left(R_{2}-R_{1}\right)}{\pi R_{2}+2 R_{2}}
$$

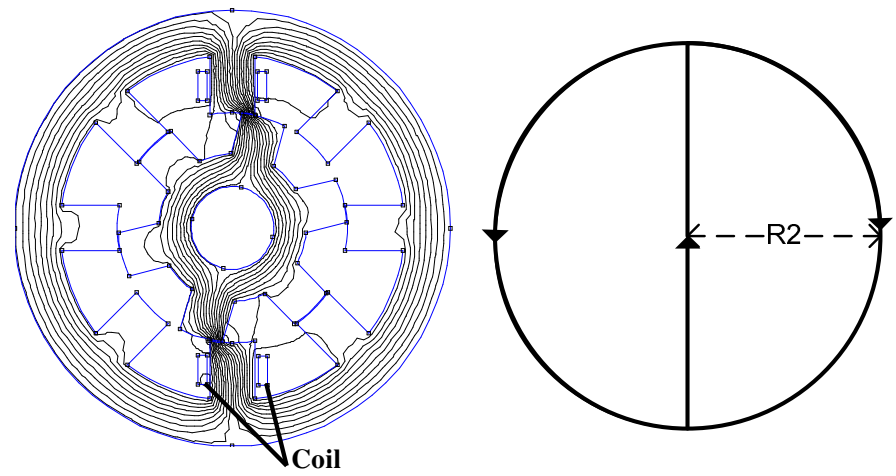

Fig4-A

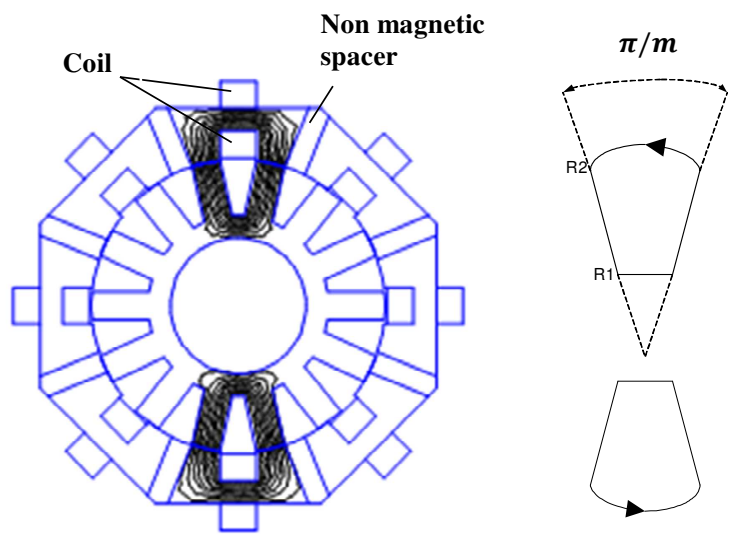

Fig4-B

Fig. 4 - A. Long flux-path for a regular topology $\left(m=4 ; N_{R}=6\right)$.

B. Short flux-path for a SPF topology $\left(m=4 ; N_{R}=14\right)$ with stator modules separated by non-magnetic spacers. 
As inferred from the geometry of both topologies, in the short flux-path case it is considered a relationship between the exterior and interior radius $\boldsymbol{R}_{\mathbf{1}}$ given by $R_{2}=3 R_{l}$. Both are four phase machines $\left(m^{\prime}=m=4\right)$ and the SFC topology has a higher number of rotor poles compared with the reference machine, $N^{\prime}{ }_{R}=14$. Taking into account the above assumptions, the ratio of fluxpath lengths $\boldsymbol{l}_{\boldsymbol{F e}, \boldsymbol{s f}} / \boldsymbol{l}_{\boldsymbol{F e}}$ is approximately $1 / 2$.

The magnetic structure that was chosen is a modular SFP topology constituted by eight stator modules separated by non-magnetic spacers. Part of the coil wound on the base of each module is exposed making the copper cooling process and the heat removal more effective. High fault tolerance, easy maintenance and simplicity of manufacturing are also favorable arguments to choose this SFP topology [33].

The laws related to differentiated scales for copper and iron provide the means to track the course of the copper losses and the material weight of the SFP topology with respect to the regular 8/6 topology. With a limited increase of temperature and a constant flux density the relationship between the iron and copper characteristic dimensions is expressed by (22) and the copper losses by (23).

$$
\begin{gathered}
B l_{F e} \propto l_{C u}{ }^{3 / 2} \\
P_{J} \propto m J^{2} l_{C u}{ }^{3} \propto m l_{C u}{ }^{2} \propto m\left(B l_{F e}\right)^{4 / 3}
\end{gathered}
$$

The copper losses depend upon the flux-path length on the core as indicated by (23).

Observing the behaviors represented in Fig. 5-A and 5-B it is further noted the decrease of the copper weight as well as the iron weight of the modular topology when compared to the reference topology. In the SFP case, the relative weight of copper is higher than the weight of iron up to approximately a rated power equal to $3 P_{N}$. Above that value the iron weight is predominant .
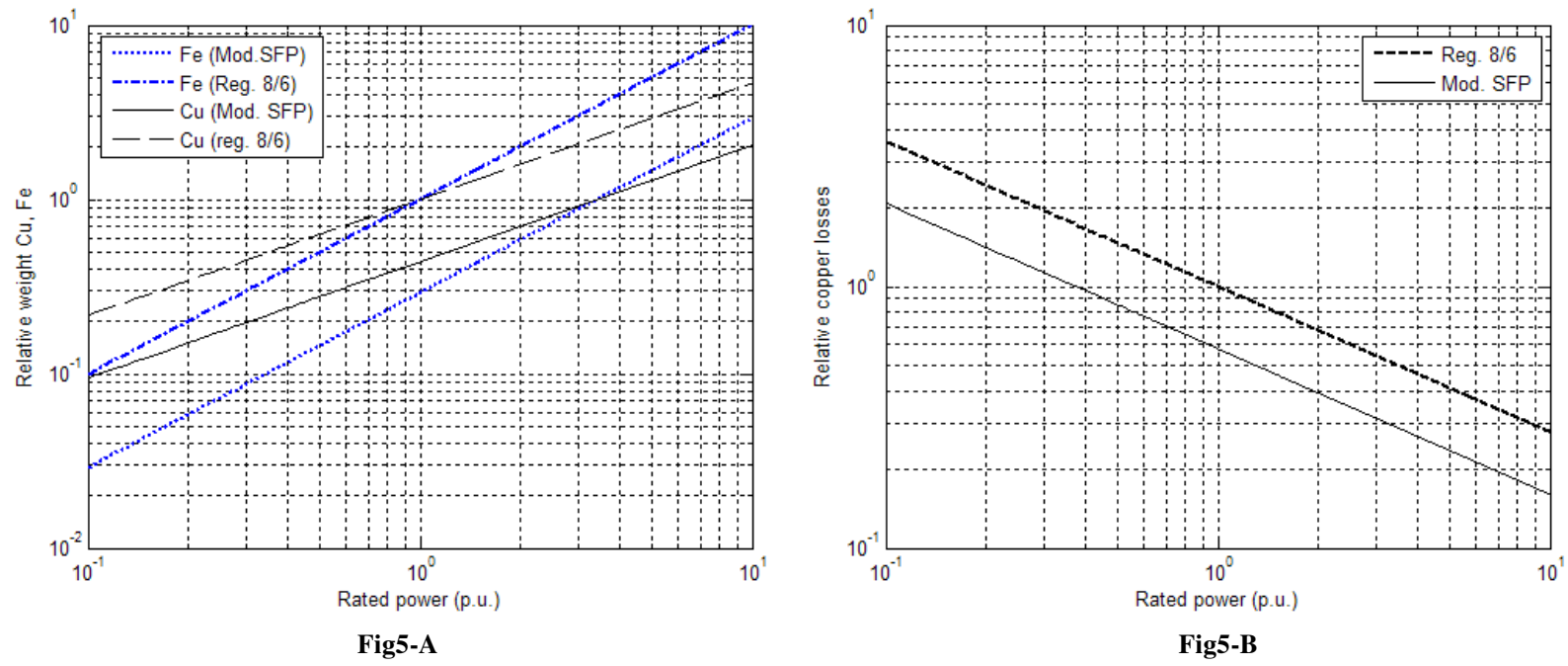

Fig. 5 - Relative weight (A) and losses (B) for SFP topology based on the regular 8/6 SRG. 
The decrease of copper losses at a large power range suggest a rescaling operation i.e. a reduction of the dimensions of the SFP machine with respect to the regular machine, considering identical rated power. For that purpose, scale laws and a simple model based on field theory will be used.

\subsection{Field-based model for dimensional analysis}

Regarding electromagnetic rotating systems and within the scope of scale comparisons, it is advantageous to work with simple models that represent the distribution of the flux density and magnetic energy. Those field-based models, supported in real system dimensions, can be used to estimate torque and power relationships. Making use of scale laws, an evaluation of relevant topology characteristics and parameters is expected to be achieved. Thereby a model of a basic reluctance rotating system will be developed. The core is built with identical and isotopic material assuming magnetic linear behavior and very high magnetic permeability.

In such SRG topologies characterized by operating into the saturation region, the idea of constructing field-based models assuming linearity of the magnetic circuit may seem contradictory. However, as the saturation effect is extended to both topologies, it is possible to compare the characteristics of two structurally different topologies (for instance, having a different number of poles) preserving certain dimensions of the magnetic circuit where the flux paths lie on. Therefore the stator exterior diameter, the air gap length $\delta_{1}$, the radius of the air gap $R_{g}$ as well as the core length $h$, will be fixed and kept constant.

Fig. 6-A shows part of a basic rotating reluctance system composed of two poles of equal dimensions one having the capability of movement (rotor pole) with respect to the other which is in a fixed position (stator pole). One of these poles is magnetized by a coil with $N$ turns carrying current $i$. Hence a torque is produced in order to reduce the reluctance of the magnetic circuit that constitutes the system, i.e. by varying the relative position of the poles.

In order to determine the position of the rotor pole and the quantities involved in the system, two angular coordinates are sufficient: $\boldsymbol{\alpha}$, the absolute coordinate linked to the fixed inertial frame of reference and $\boldsymbol{\theta}$ the relative coordinate that indicates the position of the rotor pole (rotating frame of reference) with respect to the stator pole. The rotor and stator pole arcs, $\boldsymbol{\beta}_{\boldsymbol{R}}$ and $\boldsymbol{\beta}_{S}$ are approximately equal. It is also assumed that stator winding comprises another coil, wound on a pole diametrically opposed to the first one through which the flux-path closes by itself. Adding to the above mentioned assumptions, the fringing and leakage fields in the air gap will be neglected.

Equation (1) applied to this reluctance system yields equation (24). Thus the flux density $B$ at the air gap and the magnetic energy stored in the system $W_{\delta}$, are expressed by (25) and (26). 


$$
\begin{gathered}
H_{(\alpha, \theta)} \delta_{(\alpha, \theta)}=N i \\
B_{(\alpha, \theta)}=\frac{\mu_{O} N i}{\delta_{(\alpha, \theta)}} \\
W_{\delta}=2 \int_{V_{\delta}}\left(\int_{0}^{B} H \cdot d B^{\prime}\right) d V=\int_{V_{\delta}} \frac{B^{2}}{\mu_{0}} d V
\end{gathered}
$$

It should be noted that (26) includes the presence of two volumes of air gap $V_{\delta}$ in the magnetic circuit. Regarding the air gap lengths it is assumed that $\delta_{1} \ll \delta_{2}$. In these terms, the electromagnetic torque $T_{e}$ is given by the derivative of the coenergy $W_{C}$ with respect to the rotor position (27) and the maximum torque is given by (28). The electromagnetic power in the generator regime as well as in the motor regime, shown in Fig.6-B, is calculated using the average torque $\left\langle T_{e}\right\rangle$ by (29).

$$
\begin{gathered}
T_{e}=\frac{\partial W_{C^{(i, \theta)}}}{\partial \theta}=\frac{\partial W_{\delta}(i, \theta)}{\partial \theta} \\
T=\mu_{o}(N i)^{2} h R_{g}\left(\frac{1}{\delta_{1}}-\frac{1}{\delta_{2}}\right) \cong \frac{\mu_{o}(N i)^{2} h R_{g}}{\delta_{1}} \\
P=\left\langle T_{e}\right\rangle \omega=m \frac{\beta_{R}}{\tau_{R}}\left(\frac{\mu_{o} N^{2} h R_{g}}{\delta_{1}}\right) \omega_{i_{r m s}}^{2}
\end{gathered}
$$
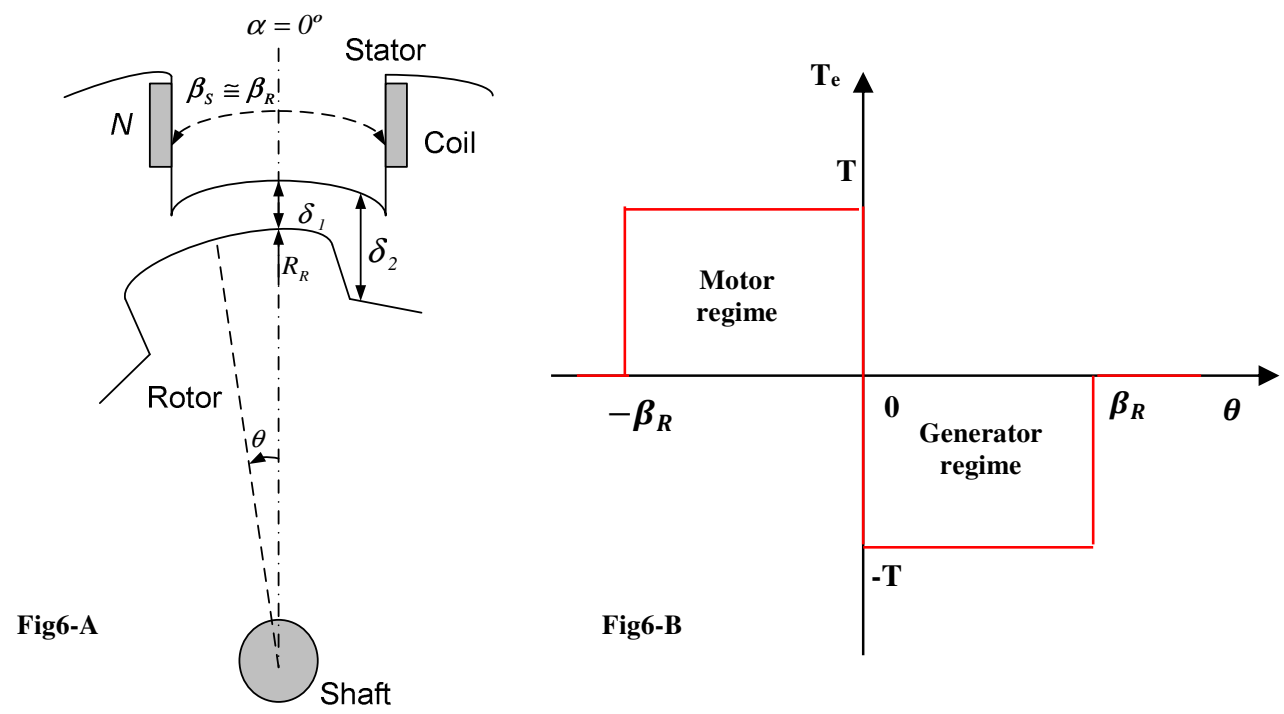

Fig. 6 - A. Schematic view of part (two poles) of a basic reluctance rotating system.

B. Torque- position profile and operation regimes of the switched reluctance system. 


\section{DESIGN STUDY RESULTS}

In this section, the scale laws methodology and the field models are used to compare the SFP topology represented in Fig.4-B with a 12/16 laboratory prototype (three-phase regular structure), built by M. A. Mueller for a direct drive wind turbine. Mueller elected this topology with 12 stator poles and 16 rotor poles based on torque density criteria [28]. However, the relevance of that work for this study lies on the similar parameters of the prototype that will support the scale comparison of topologies. In regards to these particular topologies to be compared, both magnetic circuits have four stator poles involved in the flux-path when one phase is excited. Identical air gap dimensions $\left(\boldsymbol{\delta}, \boldsymbol{R}_{\boldsymbol{g}}, \boldsymbol{L}\right)$ are assumed and an equal MMF per stator pole is imposed. Thereby equation (29) can be used. The modular SFP topology, presents a torque $56 \%$ higher than the regular machine. This added value lies in a greater number of phases (one more than the prototype) and in a larger section of rotor poles as observed in table I.

TABLE I. Parameters of the compared topologies

\begin{tabular}{c|c|c|c|c|c|} 
& $\mathbf{m}$ & $\boldsymbol{N}_{\boldsymbol{R}}$ & $\boldsymbol{\tau}_{\boldsymbol{R}}[\mathbf{r a d}]$ & $\boldsymbol{\beta}_{\boldsymbol{R}}[\mathbf{r a d}]$ & $P(p . u)$ \\
\hline $\begin{array}{c}\text { Regular 12/16 } \\
\text { prototype }\end{array}$ & 3 & 16 & $\boldsymbol{\pi} / \mathbf{8}$ & $\boldsymbol{\pi} / \mathbf{2 4}$ & 1 \\
\hline $\begin{array}{c}\text { SFP modular } \\
\text { topology }\end{array}$ & 4 & 14 & $\boldsymbol{\pi} / \mathbf{7}$ & $\boldsymbol{\pi} / \mathbf{1 8}$ & 1,56 \\
\hline
\end{tabular}

The results in terms of power allow enough flexibility to perform a rescaling operation of the modular magnetic structure.

Adopting differentiated scales, keeping the flux density and the variation temperature constant, the rated power is expressed by the relationship (30). As illustrated in the diagram of Fig. 7 and having in mind a modular machine of equal power $\left(\boldsymbol{P}_{N}^{\prime}\right)$ to the standard machine $\left(\boldsymbol{P}_{N}\right)$, the proportion of power values presented in Table I enables to infer the following rescaling relationships (31) and (32). 


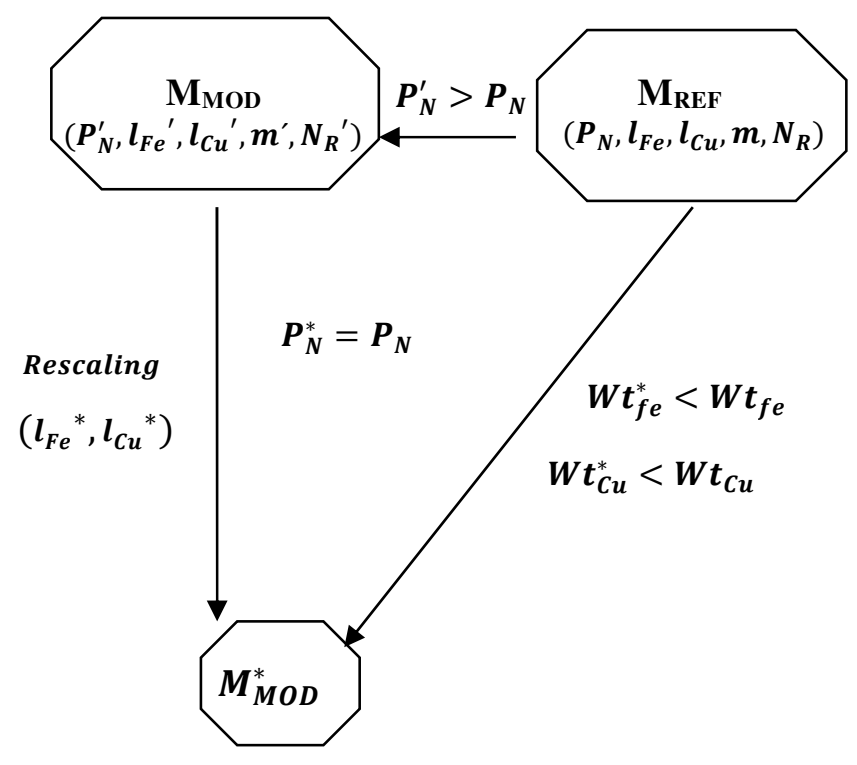

Fig. 7 - Schematic diagram of the rescaling operation for weight and losses comparison purposes.

$$
\begin{aligned}
& P_{N} \propto m N_{R} \omega B^{2} l_{F e}{ }^{3} \\
& \frac{P_{N}^{\prime}}{P_{N}} \propto \frac{m^{\prime} N_{R}^{\prime}}{m N_{R}}\left(\frac{l_{F e}}{l_{F e}}\right)^{3}
\end{aligned}
$$

$$
l_{F e}{ }^{\prime} \propto\left(\frac{1}{1,56} \frac{m N_{R}}{m^{\prime} N_{R}^{\prime}}\right)^{1 / 3} l_{F e} \propto 0,82 l_{F e}
$$

The rescaling operation shown in Fig. 7, consists of the reduction in proportional terms of the characteristic dimensions of the modular machine $\left(\mathrm{M}_{\mathrm{MOD}}\right)$ comparing to the standard machine $\left(\mathrm{M}_{\mathrm{REF}}\right)$ for identical rated power. After performing the rescaling operation, it is well-timed to establish relationships for both weight of iron $\boldsymbol{W} \boldsymbol{t}_{\boldsymbol{F e}}^{*}$ and the weight of copper $\boldsymbol{W} \boldsymbol{t}_{\boldsymbol{C} \boldsymbol{u}}^{*}$ as well as to evaluate in relative terms the copper losses $\boldsymbol{P}_{J \boldsymbol{r}}^{*}$. The iron weight and the copper weight of the modular topology are compared with the SRG prototype as indicated by (33) and (34). 


$$
\begin{gathered}
W t_{F e}^{*} \propto \frac{\left(l_{F e}{ }^{*}\right)^{3}}{\left(l_{F e}\right)^{3}} \propto 0,55 W t_{F e} \\
\mathbf{W t}_{\mathrm{Cu}}^{*} \propto \frac{\left(\mathbf{l}_{\mathrm{Cu}}{ }^{*}\right)^{3}}{\left(\mathbf{l}_{\mathrm{Cu}}\right)^{3}} \mathbf{W t}_{\mathrm{Cu}} \propto \frac{\left(\mathbf{l}_{\mathrm{Fe}}{ }^{*}\right)^{2}}{\left(\mathbf{l}_{\mathrm{Fe}}\right)^{2}} \mathbf{W t}_{\mathrm{Cu}} \propto 0,67 \mathrm{Wt}_{\mathrm{Cu}}
\end{gathered}
$$

In regards to the modular topology, the iron weight is approximately $55 \%$ of the standard topology, thus reducing the volume taken by the iron by $45 \%$. In terms of copper weight it allows a reduction of $33 \%$ in respect to the regular $12 / 16$ SRG.

In terms of specific power, expressed in $\mathrm{W} / \mathrm{Kg}$, it is predicted that there will be an increase of power of $80 \%$ at the modular SRG per unit of iron mass and an increase of close to $50 \%$ per unit of copper mass when compared to the standard SRG.

According to (35) and (36), even after the rescaling operation and the resultant reduction of the modular machine volume, the relative losses of both machines show the same proportionality. The rescaling operation is performed on an identical rated power basis.

$$
\begin{gathered}
\frac{\boldsymbol{P}_{J r}^{*}}{\boldsymbol{P}_{J r}}=\frac{\frac{\boldsymbol{P}_{J}^{*}}{\boldsymbol{P}_{N}^{*}}}{\frac{\boldsymbol{P}_{J}}{\boldsymbol{P}_{N}}} \propto \frac{m^{*}\left(\boldsymbol{l}_{C u}{ }^{*}\right)^{2}}{m \boldsymbol{l}_{C u}{ }^{2}} \propto 1, \quad \boldsymbol{P}_{N}^{*}=\boldsymbol{P}_{N} \\
\boldsymbol{P}_{J r}^{*} \propto \boldsymbol{P}_{J r}
\end{gathered}
$$

Due to the general high altitude location of the wind turbines, whether onshore or offshore, the weight of the materials might be a decisive factor in choosing the equipment to be used as generator. Together with lesser weight comes lower cost of material and maintenance. Furthermore, higher fault tolerance is expected when the modular topology is selected. The heat removal and the temperature distributed within the machine benefit from the modular configuration and the winding location on the stator modules. In fact the heat transfer is at least as determining as the electromagnetic design and will be treated in a future work.

Finally, good performance is preserved and it is not expected that the inclusion of iron losses in the previous calculations will degrade results in a significant way. 


\section{CONCLUSIONS}

This paper has presented a SRG design methodology, based on scale models for low speed applications. This work is motivated by wind and other renewable energy systems which are characterized by a low rotational speed. General design methodologies are usually oriented towards the choice of stator/rotor poles combinations in regular topologies. Besides covering that feature, the scale methodology under consideration is able to compare different magnetic topologies, regular or non-regular, and also has the capability to incorporate thermal and magnetic saturation phenomena by introducing constraints. Therefore, this paper extends previous discussions of SRG design into a more general context.

Based on the similarity laws and dimensional analysis, a SRG modular topology was selected and compared with a prototype rated at $20 \mathrm{~kW}, 100 \mathrm{rpm}$ designed for a wind energy converter. The comparison results point out the significant gain of power per unit of volume and the reduction of losses of the modular topology over the prototype. The methodology proposed is also applicable to the design of low-speed SR motors and can be used over a wide speed range.

This work is not intended to be a detailed design of a novel SRG neither an original methodology but rather indicative of the importance of the scale models formulation as an assistance tool for this machine design. Therefore, its application to different SR topologies clearly emphasizes some design details of magnetic structures that will enhance machine performance. Ultimately this work attempts to expand the discussion on SRG design for low speed applications, usually confined to regular topologies, in a broader context that includes other constructive paradigms, by changing electric and magnetic circuits and their own relative location. Moreover, it sets out a platform for further discussion.

As a guideline, and not as an end, the comparison results achieved in this dimensional analysis indicate that additional work should be developed concerning a detailed design of the modular SFP topology. Taking into account real dimensions and material characteristics, a full scale machine design will make it possible to compare initial costs of the modular SFP SRG topology with classic generators using gearbox.

\section{ACKNOWLEDGEMENT}

The author would like to thank the Polytechnic Institute of Setúbal and FCT /New University of Lisbon for providing facilities as part of an existing cooperation protocol. 


\section{REFERENCES}

[1] I. Boldea, L.N. Tutelea, L. Parsa, D. Dorrell, "Automotive Electric Propulsion Systems With Reduced or No Permanent Magnets: An Overview", IEEE Transactions on Industrial Electronics, vol.61, no.10, pp.5696-5711, Oct. 2014

[2] C. Sahin, A.E. Amac, M. Karacor, A. Emadi, "Reducing torque ripple of switched reluctance machines by relocation of rotor moulding clinches", IET Electric Power Applications, vol.6, no.9, pp.753-760, November 2012

[3] Hao Chen, J.J. Gu, "Implementation of the Three-Phase Switched Reluctance Machine System for Motors and Generators", IEEE/ASME Transactions on Mechatronics, vol.15, no.3, pp.421-432, June 2010

[4] Wen Ding, Ling Liu, Jianyong Lou, "Design and control of a high-speed switched reluctance machine with conical magnetic bearings for aircraft application", IET Electric Power Applications, vol.7, no.3, pp.179-190, March 2013

[5] Yihua $\mathrm{Hu}$; Xueguan Song; Wenping Cao; Bing Ji, "New SR Drive With Integrated Charging Capacity for Plug-In Hybrid Electric Vehicles (PHEVs)", IEEE Transactions on Industrial Electronics , vol.61, no.10, pp.5722-5731, Oct. 2014

[6] R. Vandana, S. Nikam, B.G. Fernandes, "High torque polyphase segmented switched reluctance motor with novel excitation strategy", IET Electric Power Applications, vol.6, no.7, pp.375-384, August 2012

[7] K.F. Wong, K.W.E. Cheng, S.L. Ho, "Four-quadrant instantaneous torque control of switched reluctance machine at low speed based on co-energy control", IET Electric Power Applications, vol.3, no.5, pp.431-444, September 2009

[8] V. Rallabandi, B.G. Fernandes, "Design procedure of segmented rotor switched reluctance motor for direct drive applications", IET Electric Power Applications, vol.8, no.3, pp.7788, March 2014

[9] Yan-Tai Chang; K.W.E. Cheng, "Sensorless position estimation of switched reluctance motor at startup using quadratic polynomial regression", IET Electric Power Applications, vol.7, no.7, pp.618-626, Aug. 2013

[10] P. Andrada, B. Blanqué, E. MartíNez, J.I. Perat, J.A. Sánchez, M. Torrent, "Environmental and life cycle cost analysis of one switched reluctance motor drive and two inverter-fed induction motor drives", IET Electric Power Applications, vol.6, no.7, pp.390-398, August 2012 
[11] A. Siadatan, E. Afjei, H. Torkaman, M. Rafie, "Design, simulation and experimental results for a novel type of two-layer 6/4 three-phase switched reluctance motor/generator", Energy Conversion and Management, vol.71, pp. 199-207,July 2013.

[12] V. Valdivia, R. Todd, F.J. Bryan, A. Barrado, A. Lazaro, A.J. Forsyth, "Behavioral Modeling of a Switched Reluctance Generator for Aircraft Power Systems", IEEE Transactions on Industrial Electronics, vol.61, no.6, pp.2690-2699, June 2014

[13] Y.J. Bao, K.W.E. Cheng, N.C. Cheung, S.L. Ho , "Experimental examination on a new switched reluctance wind power generator system for electric vehicles", IET Power Eletronics, vol. 5, Issue 8, pp. 1262-1269, Sept. 2012.

[14] C.A. Ferreira, S.R. Jones, W.S. Heglund, Jones W.D., "Detailed design of a 30-kW Starter/Generator System for a Gas turbine Engine Application”, IEEE Trans. on Industry Applications, vol. 31, no.3, pp. 553-561, May/June 1995.

[15] Hany M. Hasanien, S.M. Muyeen, "Speed control of grid-connected switched reluctance generator driven by variable speed wind turbine using adaptive neural network controller", Electric Power Systems Research, vol 84, Issue 1, pp. 206-213, March 2012.

[16] B. Fahimi, A.R.B. Emadi, Jr. Sepe, "A Switched Reluctance Machine Based Starter/Alternator for more Electric Cars", IEEE Trans. on Energy Conversion, vol. 19, pp. 116-124, Mar. 2004.

[17] H. Yahia, N. Liouane, R. Dhifaoui, "Differential evolution method-based output power optimisation of switched reluctance generator for wind turbine applications", IET Renewable Power Generation, vol. 8, Issue 7, pp.795-806, Sept. 2014.

[18] P. Chancharoensook, M.F. Rahman, "Control of a four-phase switched reluctance generator: experimental investigations", Proc. of the IEEE International Electric Machines and Drives Conference, vol.2, pp.842-848, June 2003.

[19] T.J.E Miller, Switched Reluctance Motors and Their Control, Magna Physics Publishing and Clarendon Press, 1993.

[20] P. Lobato, J. Martins, A.J. Pires, "A design criteria for torque ripple reduction in Switched Reluctance Generators", Proc. of 2011 International Conference on Power Engineering, Energy and Electrical Drives (POWERENG), pp.1-6, May 2011.

[21] I. Kioskeridis, C. Mademlis., "Optimal Efficiency Control of Switched Reluctance Generators”, IEEE Trans. on Power Electronics, vol. 21, No.4, July 2006.

[22] R. Cardenas , R. Pena , M. Perez , J. Clare , G. Asher and P. Wheeler, "Control of a switched reluctance generator for variable-speed wind energy applications", IEEE Trans. on Energy Conversion, vol. 20, no. 4, pp.781 -791, 2005. 
[23] B. Bilgin, A. Emadi, M. Krishnamurthy, "Design Considerations for Switched Reluctance Machines With a Higher Number of Rotor Poles", IEEE Trans. on Industrial Electronics, vol.59, no.10, pp.3745-3756, Oct. 2012.

[24] K.M. Rahman, S.E. Schulz, "Design of high-efficiency and high-torque-density switched reluctance motor for vehicle propulsion", IEEE Trans. on Industry Applications, vol.38, no.6, pp.1500-1507, Nov/Dec 2002.

[25] K.M. Rahman, B. Fahimi, G. Suresh, A.V. Rajarathnam, M. Ehsani, "Advantages of switched reluctance motor applications to EV and HEV: design and control issues", IEEE Trans. on Industry Applications, vol.36, no.1, pp.111-121, Jan/Feb 2000.

[26] S.M. Lukic, A. Emadi, "State-Switching Control Technique for Switched Reluctance Motor Drives: Theory and Implementation", IEEE Trans. on Industrial Electronics, vol.57, no.9, pp.2932-2938, Sept. 2010.

[27] D.A. Torrey, M. Hassanin, "The design of low-speed variable-reluctance generators", Conference Record of the 1995 IEEE Industry Applications Conference, vol.1, pp.427-433, 8-12 Oct. 1995.

[28] M.A. Mueller, "Design and performance of a $20 \mathrm{~kW}, 100 \mathrm{rpm}$, switched reluctance generator for a direct drive wind energy converter", Proc. of the IEEE International Conference on Electric Machines and Drives, San Antonio, Texas, U.S.A., pp. 56-63, 2005.

[29] Marcel Jufer, "Electric Drive: Design Methodology”, Wiley-ISTE press, 2013.

[30] P.N. Materu, R. Krishnan "Estimation of switched reluctance motor losses", IEEE Trans. on Industry Applications, vol.28, no.3, pp.668-679, May/Jun 1992.

[31] B. Parreira, S. Rafael, A. J. Pires, P. J. Costa Branco, "Obtaining the magnetic characteristics of an 8/6 switched reluctance machine: FEM analysis and experimental tests", IEEE Transactions on Industrial Electronics, vol.52, no.6, pp. 1635-1643, Dec. 2005.

[32] L. Szabó, M. Ruba, "Segmental Stator Switched Reluctance Machine for Safety-Critical Applications," IEEE Transactions on Industry Applications, vol.48, no.6, pp.2223-2229, Nov/Dec. 2012

[33] M. Ruba, I. Viorel, L. Szabó, "Modular stator switched reluctance motor for fault tolerant drive systems", IET Electric Power Applications, vol. 7, Issue 3, pp. 159-169, March 2013. 